\title{
Delivered dose with jet and mesh nebulisers during spontaneous breathing, noninvasive ventilation and mechanical ventilation using adult lung models
}

\author{
Arzu Ari (1) and James B. Fink
}

Dept of Respiratory Care, Texas State University, Round Rock, TX, USA.

Corresponding author: Arzu Ari (E-mail: arzuari@txstate.edu)

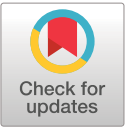

This version is distributed under the terms of the Creative Commons Attribution NonCommercial Licence 4.0. For commercial reproduction rights and permissions contact permissions@ersnet.org

Received: 13 Jan 2021 Accepted: 20 April 2021

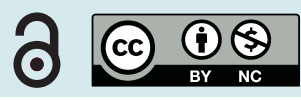

Shareable abstract (@ERSpublications)

Delivered dose distal to the trachea of an adult lung model is similar or greater with MV than NIV and SB, suggesting that the artificial airway may provide a similar or better pathway for medical aerosols to the lung https://bit.ly/3gAk7nd

Cite this article as: Ari A, Fink JB. Delivered dose with jet and mesh nebulisers during spontaneous breathing, noninvasive ventilation and mechanical ventilation using adult lung models. ERJ Open Res 2021; 7: 00027-2021 [DOI: 10.1183/23120541.00027-2021].

\section{Abstract}

What is the delivered dose with jet and mesh nebulisers during spontaneous breathing (SB), noninvasive ventilation (NIV), and mechanical ventilation (MV) using an adult lung model with exhaled humidity (EH)?

The delivery of salbutamol sulfate $(2.5 \mathrm{mg}$ per $3 \mathrm{~mL}$ ) with jet (Mistymax10) and mesh nebulisers (Aerogen Solo) was compared during SB, NIV, and MV using breathing parameters (tidal volume $450 \mathrm{~mL}$, respiratory rate 20 breaths per min, inspiratory:expiratory ratio 1:3) with three lung models simulating exhaled humidity. A manikin was attached to a sinusoidal pump via a filter at the bronchi to simulate an adult with SB. A ventilator (V60) was attached via a facemask to a manikin with a filter at the bronchi connected to a test lung to simulate an adult receiving NIV. A ventilator-dependent adult was simulated through a ventilator (Servo-i) operated with a heated humidifier (Fisher \& Paykel) attached to an endotracheal tube (ETT) with a heated-wire circuit. The ETT was inserted into a filter (Respirgard II). A heated humidifier was placed between the filter and test lung to simulate exhaled humidity $\left(35 \pm 2^{\circ} \mathrm{C}, 100 \%\right.$ relative humidity). Nebulisers were placed at the Y-piece of the inspiratory limb during MV and positioned between the facemask and the leak-port during NIV. A mouthpiece was used during SB. The delivered dose was collected in an absolute filter that was attached to the bronchi of the mannequin during each aerosol treatment and measured with spectrophotometry.

Drug delivery during MV was significantly greater than during NIV and SB with a mesh nebuliser $(p=0.0001)$ but not with a jet nebuliser $(p=0.384)$. Delivery efficiency of the mesh nebuliser was greater than the jet nebuliser during MV ( $p=0.0001)$, NIV ( $p=0.0001)$, and SB ( $p=0.0001)$.

Aerosol deposition obtained with a mesh nebuliser was greater and differed between MV, NIV, and SB, while deposition was low with a jet nebuliser and similar between the modes of ventilation tested.

\section{Introduction}

Aerosolised medications are commonly used in the treatment of patients with pulmonary diseases. For many decades, researchers have attempted to understand variables impacting aerosol drug delivery with different nebulisers and levels of ventilatory support [1-21]. However, little has been done to identify the best methods of delivering aerosolised medications to different patient populations such as ventilator-dependent patients, spontaneously breathing adults, and patients receiving noninvasive ventilation (NIV). Assumptions are commonly made that patients with spontaneous breathing (SB) inherently receive more drugs than patients receiving ventilatory support. One rationale has been that there is a greater aerosol loss in artificial airways or breathing through the mask than through the mouthpiece. Also, the clinical decision to optimise aerosol delivery is often arbitrary, and the exact drug dosages are used with different nebulisers and patient populations. With increasing recognition of inefficient aerosol 
therapy and its consequences in healthcare, identifying strategies to optimise aerosol delivery with various nebulisers in different patient populations remains a high priority.

MAcInTyre et al. [22] reported radiolabelled aerosol deposition with jet nebulisers of $12 \%$ via a mouthpiece with SB subjects compared with 3\% via an endotracheal tube (ETT) during mechanical ventilation (MV) with a heated humidifier. Since this seminal paper in 1985, the assumption has been that inhaled dose is greater with SB than MV, especially with jet nebulisers. The lower dose with MV was due to aerosol passing through the ETT.

Since that time, there have been no reports of direct comparison of aerosol delivery efficiency with MV, NIV, and SB under similar breathing parameters. Although previous research has found many factors that affect aerosol delivery to patients, no single approach has been determined as the best one. Also, many patients transition between spontaneous breathing, NIV, and MV. Although aerosol therapy is undertaken based on the clinician's judgment and experience, it is not individualised based on the patient's needs and the aerosol device used for therapy. In patients receiving aerosol therapy, there is a tendency to apply the same dose across interfaces. While the same drug dosages are used for all patient populations and nebulisers, there are no reports that compared the delivery efficiency of jet and mesh nebulisers between various modes of ventilation. Also, previous in vitro studies reported up to a $40 \%$ reduction in aerosol drug delivery with heated humidified ventilator circuits [23-26]. However, the lung models with dry or heated/humified circuits that were used in these studies do not simulate active heating and humidity of exhaled gases. Whether the patient is ventilator dependent or spontaneously breathing, they exhale heated and humidified gas close to body temperature. Our previous work showed that in vitro lung models without exhaled humidity overestimate aerosol drug delivery compared with models using exhaled humidity in simulated spontaneously breathing tracheotomised patients, as well as simulated ventilator-dependent patients using heat moisture exchangers (HMEs) [2-4, 27]. However, to date, there is no study that evaluates the impact of exhaled humidity on aerosol drug delivery across different types of ventilation. Therefore, the purpose of this study is to quantify and compare aerosol deposition with jet and mesh nebulisers during SB, NIV, and MV using adult lung models with exhaled humidity. Understanding differences in delivery efficiency between MV, NIV, and SB could provide guidance as to how to adjust doses between types of ventilation to improve consistent targeted drug delivery to the lungs of patients.

\section{Materials and methods}

\section{Study design}

As shown in figure 1, drug delivery with jet (Mistymax10; Carefusion, Yorba Linda, CA, USA) and mesh nebuliser (Aerogen Solo; Aerogen Ltd., Galway, Ireland) was compared during SB, NIV, and MV using three different lung models with exhaled humidity.

\section{Lung models}

To simulate a mechanically ventilated adult, a ventilator (Servo-I; Getinge, Wayne, NJ, USA) was operated with a heated humidifier (Fisher \& Paykel Healthcare, Auckland, New Zealand) attached to an $8 \mathrm{~mm}$ internal diameter (ID) ETT via a heated-wire circuit (figure 2a). The ETT cuff was inflated in a $15 \mathrm{~mm}$ ID/

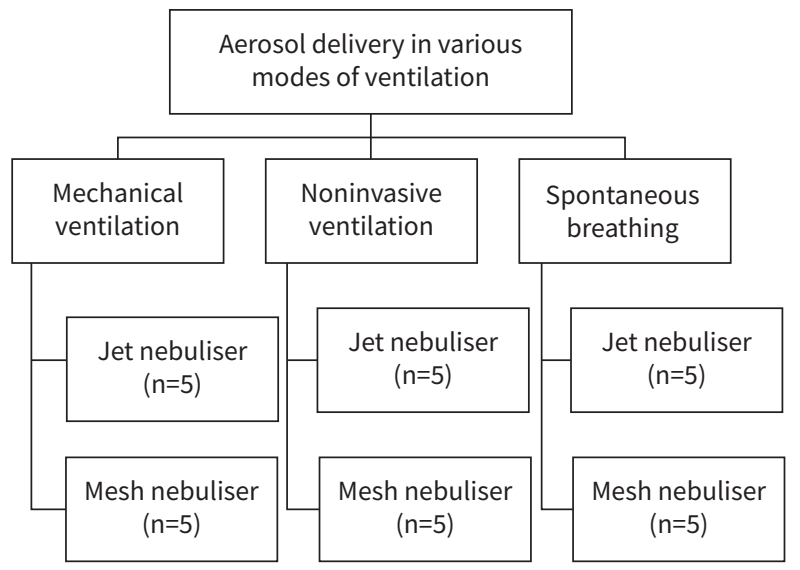

FIGURE 1 Schematic of the study design, including each nebuliser and modes of ventilation. 


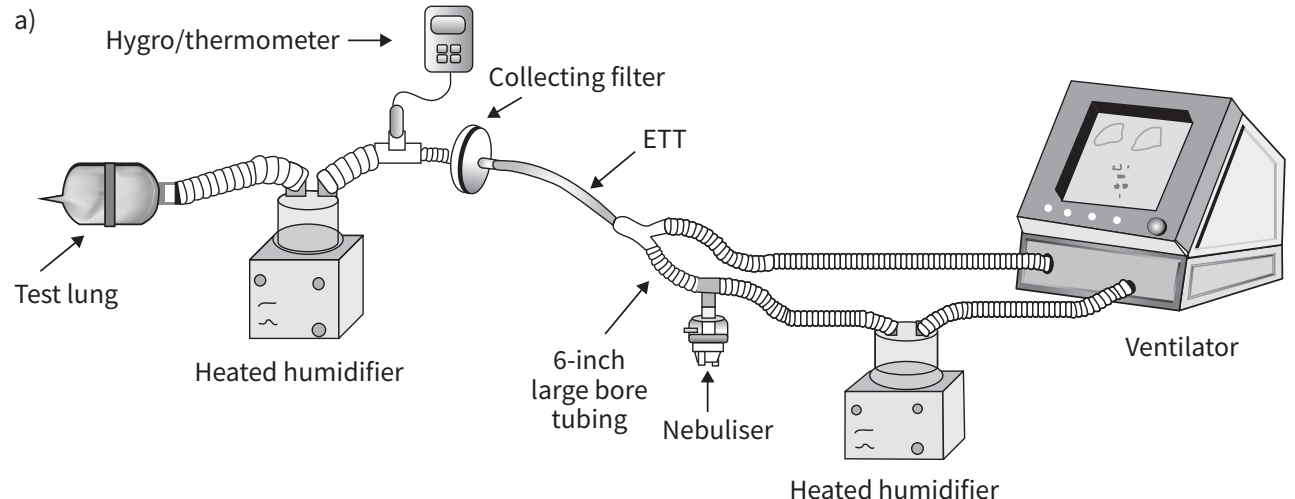

b)

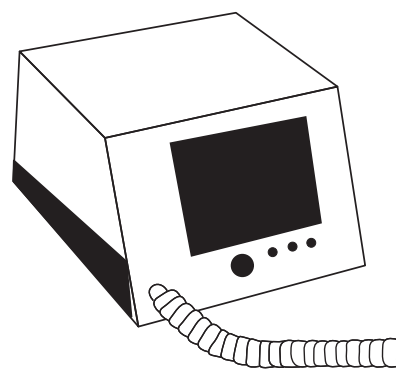

Noninvasive ventilator

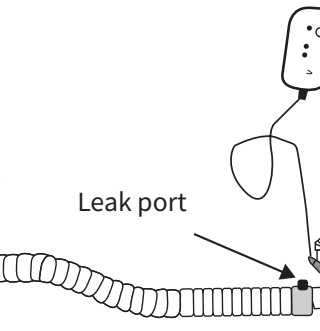

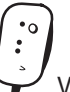

Vibrating mesh nebuliser

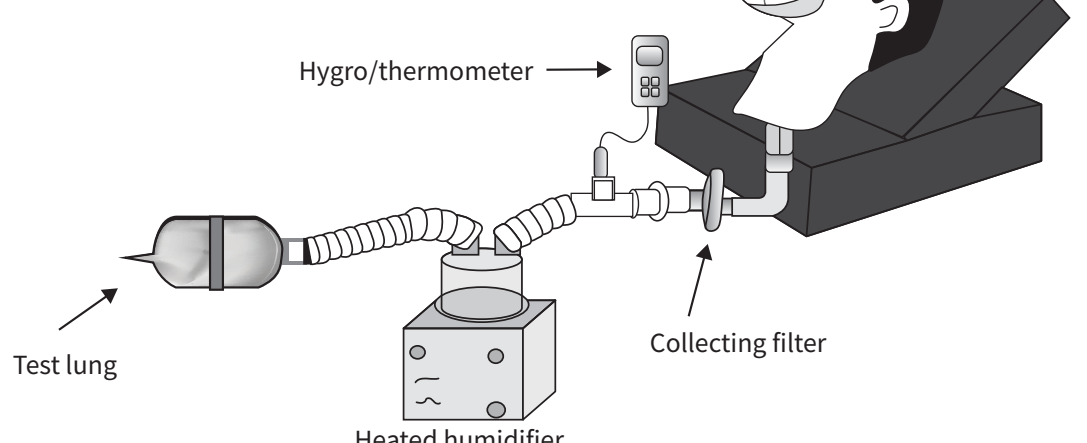

c)

Heated humidifier

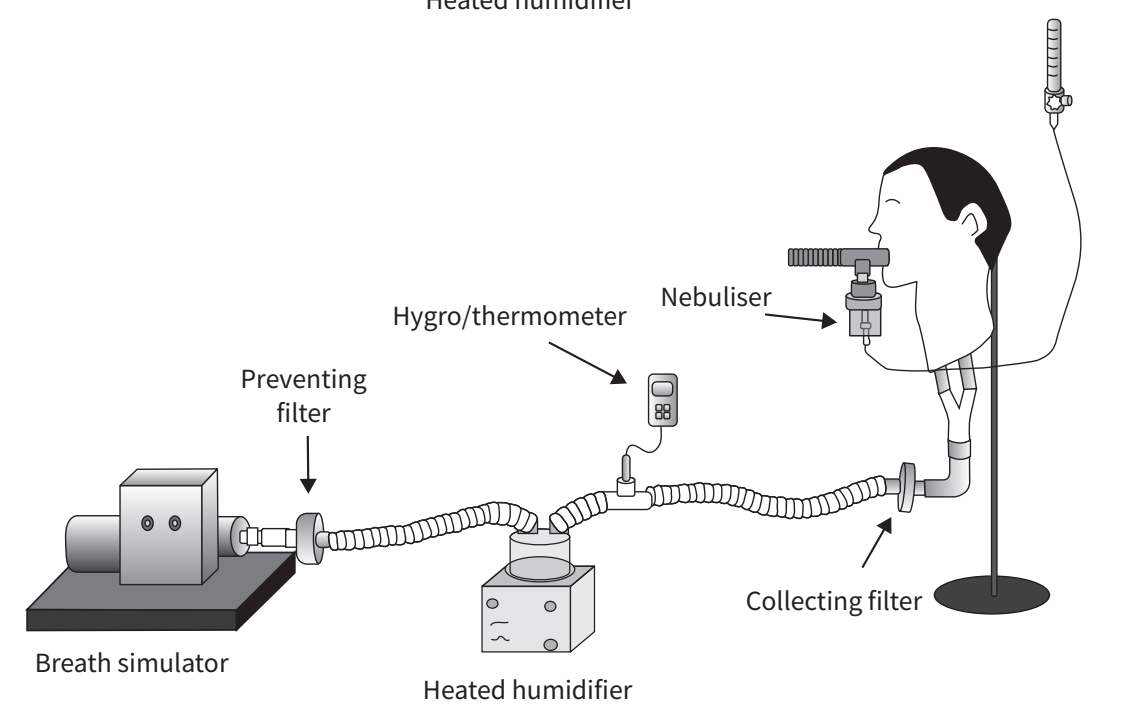

FIGURE 2 Lung models used in this study. a) Mechanical ventilation lung model, b) noninvasive ventilation lung model, and c) spontaneous breathing lung model. ETT: endotracheal tube. 
$22 \mathrm{~mm}$ outer diameter (OD) adapter, which was then inserted into the housing of an absolute filter (Respirgard II; Vital Signs, Totowa, NJ, USA), fixing the tip of the ETT $\sim 1 \mathrm{~cm}$ from the filter media. A heated humidifier (Fisher \& Paykel) was placed between the collecting filter and test lung to simulate exhaled humidity $\left(35 \pm 2{ }^{\circ} \mathrm{C}, 100 \%\right.$ relative humidity) as with all lung models used in this study, with exhaled temperature and humidity measured with a Digital hygrometer/thermometer (Model 485; Dwyer, Michigan City, IN, USA) between the humidifier and collecting filter. Breathing parameters were set at tidal volume $450 \mathrm{~mL}$, respiratory rate 20 breaths per min, inspiratory:expiratory ratio 1:3. Figure 2 shows the lung models used in this study.

As shown in figure 2b, an adult receiving NIV was simulated using a turbine ventilator (V60 Philips Healthcare, New Jersey, USA) with an unheated single-limb circuit incorporating a fixed leak that was attached via a nonvented oronasal face mask (AF541; Respironics Inc, Murrysville, PA, USA). The mask was securely attached to the face of an adult teaching manikin, with a collecting filter placed at the level of the bronchi distal to the trachea and connected to a passive test lung via a heated humidifier (Fisher \& Paykel Healthcare).

To simulate a spontaneously breathing adult, a teaching manikin with a collecting filter was placed at the level of the bronchi and connected via a heated humidifier (Fisher \& Paykel) to a breathing simulator (figure 2c).

\section{Data collection and analysis}

During MV, aerosol devices were placed in the inspiratory limb proximal to the Y-piece with a T-piece provided by the manufacturers, while all aerosol devices were positioned at the patient end of the single-limb circuit between the fixed leak port and face mask during NIV. In SB, a mouthpiece was sealed to the mouth of the mannequin, and the nares were sealed during aerosol therapy. The jet nebuliser was used with a simple T-piece provided by the manufacturer, with a 6-inch length of $22 \mathrm{~mm}$ ID corrugated tubing attached to the other end of the T-piece, serving as a reservoir. The mesh nebuliser was attached to a valved chamber (Aerogen Ultra; Aerogen Ltd, Galway, Ireland) with supplementary oxygen of $6 \mathrm{~L} \cdot \mathrm{min}^{-1}$. The jet nebuliser was also operated at $6 \mathrm{~L} \cdot \mathrm{min}^{-1}$ with oxygen continuously until sputter, while the mesh nebuliser was run until the end of nebulisation. The condensation in the circuits was cleared before each run, and the lung model was stabilised for a few minutes before the start of each experiment.

Salbutamol sulfate $(2.5 \mathrm{mg}$ per $3 \mathrm{~mL}$ ) was placed into the reservoir with both the jet and mesh nebuliser, and each experiment was run five times $(n=5)$. The delivered dose was collected in an absolute filter that was attached to the bronchi of the mannequin during each aerosol treatment and measured with spectrophotometry. On completion of each experiment, the collecting filters were capped and labelled for analysis. The drug deposited on an absolute filter was eluted with $10 \mathrm{~mL}$ solution (20\% ethanol with $0.1 \mathrm{~N}$ $\mathrm{HCl}$ ). The amount of salbutamol sulfate depositing in the collecting filter was analysed via spectrophotometry $(276 \mathrm{~nm})$ and expressed as the total fraction of the nominal dose placed in each nebuliser. Descriptive statistics were used to calculate the means and standard deviations for each component of the total inhaled drug mass obtained with the jet and mesh nebuliser during SB, NIV, and MV. Differences in means between the inhaled mass for the two nebulisers and three modes of ventilation were compared with a two-way factorial analysis of variance. Paired t-test was used to compare jet nebuliser and mesh nebuliser at each mode of ventilation. When the p-value was $<0.05$, differences were considered statistically significant.

\section{Results}

\section{Effect of modes of ventilation on aerosol deposition}

Table 1 shows the percentage of the salbutamol sulfate dose (mean \pm sD) delivered distal to the trachea airway of the models with the jet and mesh nebuliser during MV, NIV, and SB in adults. Regardless of the type of nebuliser used, aerosol drug delivery during MV trended higher than NIV and SB. Aerosol delivery with a

\begin{tabular}{|c|c|c|c|c|}
\hline & Mechanical ventilation & Noninvasive ventilation & Spontaneous breathing & p-value \\
\hline Jet nebuliser & $6.80 \pm 1.55$ & $6.10 \pm 0.34$ & $6.12 \pm 0.26$ & 0.384 \\
\hline Mesh nebuliser & $23.16 \pm 0.67$ & $18.36 \pm 1.06$ & $17.29 \pm 1.69$ & 0.0001 \\
\hline p-value & 0.0001 & 0.0001 & 0.0001 & \\
\hline
\end{tabular}


mesh nebuliser was greater with MV than with NIV or SB ( $p=0.0001)$; however, there was no significant difference in aerosol delivery with mesh nebuliser between NIV and SB ( $p=0.411)$. In contrast, no difference between the three modes of ventilation was found when the jet nebuliser was used in this study ( $\mathrm{p}=0.384)$.

Effect of nebulisers on aerosol deposition

As shown in table 1, the delivery efficiency of the mesh nebuliser was greater than the jet nebuliser during MV ( $p=0.0001)$, NIV ( $p=0.0001)$, and SB ( $p=0.0001)$. Regardless of the mode of ventilation tested in this study, using a mesh nebuliser for aerosol therapy increased drug delivery $>3$-fold compared with the jet nebuliser.

\section{Discussion}

In this study, we simulated identical adult breathing parameters during aerosol administration with MV, NIV, and SB to compare inhaled dose distal to the trachea with both jet and mesh nebulisers. This is the first study to show that aerosol delivery via MV with adult breathing parameters was greater than or equal to NIV and SB with both jet and mesh nebulisers, with similar delivery efficiency between NIV and SB.

Our results are consistent with prior reports, with delivery efficiency of a mesh nebuliser up to three-fold greater than a jet nebuliser during MV, NIV, and SB. In our previous in vitro research on the influence of nebuliser type in a simulated adult lung model during mechanical ventilation [28], the inhaled dose obtained with the jet nebuliser was $4.7 \%$ as opposed to $5.2 \%$ in this study. Aerosol delivery with the mesh nebuliser was $13.4 \%$ in our previous research in contrast to $23 \%$ in this study. The discrepancy in our findings can be explained by the differences in our models and peak inspiratory flow rates (PIF) we used in both studies. In the current study, we used two heated humidifiers with the lung model. While one was used to simulate exhaled humidity, the other one provided heated humidity during MV. The PIF was set at $60 \mathrm{~L} \cdot \mathrm{min}^{-1}$ in our previous research, compared to the lower rate of $45 \mathrm{~L} \cdot \mathrm{min}^{-1}$ in this study.

Using a similar ventilator-dependent adult lung model with exhaled humidity, our previous research evaluated the effect of HMEs on aerosol deposition during MV [2]. The findings of this study showed that aerosol delivery with a mesh nebuliser was $10.6 \%$ as opposed to $5.4 \%$ with jet nebuliser in the control group, where no heated humidifier was used in the circuit. Also, the placement of HME between the patient and the nebuliser reduced aerosol deposition by $5.91 \%$ and $2.80 \%$ with the mesh nebuliser and jet nebuliser, respectively. In our current study, we used a heated humidifier in the ventilator circuit, this explains at least part of the differences in our results.

ALQuAimi et al. [5] determined the efficiency of jet and mesh nebulisers during NIV. Using an in vitro lung model without exhaled humidity, they reported an aerosol deposition of $13 \%$ and $29 \%$ with jet and mesh nebulisers, respectively. In contrast, our results showed lower delivery efficiency with both the jet nebuliser (6\%) and mesh nebuliser (18\%). The difference again may reside in the exhaled humidity simulated with our current model. Regardless of the type of nebuliser used, aerosol delivery during NIV was higher than with the exhaled humidity model used in this research. In each iteration of the model, exhaled gas was heated to simulate gas conditions leaving the lungs. This has been shown to reduce some of the higher inhaled doses previously reported with models that use unconditioned exhaled gas [12, 23, 24, 26, 29].

MAcInTYRE et al. [22] used radiolabelled aerosol with a jet nebuliser and reported $12 \%$ lung deposition via a mouthpiece with SB compared with 3\% in intubated subjects receiving MV with a heated humidifier. Since this seminal paper in 1985, the assumption has been that the inhaled dose is greater with SB than MV, especially with a jet nebuliser. The lead author (personal communication) posited the lower dose with MV to aerosol passing through the ETT. In our study, the ETT may provide a similar to or better pathway for aerosol than the upper airway model. In their study, MacInTYRE et al. [22] placed the nebuliser in the inspiratory limb of the ventilator circuit. A previous study with similar adult parameters and placement of the nebuliser in the inspiratory limb of a heated humidified circuit reported a delivered dose of $3.61 \pm 0.2 \%$ with the jet nebuliser [12]. Based on the lower PIF in our current study, we would expect a higher inhaled dose with the jet nebuliser consistent with in vitro model reported by DugERNIER et al. [30].

Galindo-Filho et al. [31] evaluated aerosol drug delivery during NIV in patients with asthma exacerbations. They reported no improvement in a radio-aerosol pulmonary deposition during NIV compared with the control group that had nebulisation alone. This is consistent with our findings of no significant difference in aerosol deposition between NIV and SB.

More recently, Galindo-FiLho et al. [32], comparing the delivery of radiolabelled aerosol during NIV with jet and mesh nebulisers in moderate-to-severe COPD, patient-reported lung doses of $3.14 \%$ with a jet 
nebuliser versus $12.05 \%$ with a mesh nebuliser. Their lower reported lung dose than our in vitro reports is probably due to our use of collecting filters that are known to overestimate inhaled dose, and filters do not allow for exhalation of aerosol that occurs in vivo. However, during NIV, aerosol deposition with a mesh nebuliser was $>3$-fold greater than jet nebuliser in both in vivo and in vitro reports.

In a radio-aerosol scintigraphy study, FrancA et al. [33] compared pulmonary deposition with a jet nebuliser in 13 healthy subjects. They reported lower lung deposition during NIV compared with SB. However, their measures of respiratory rate, tidal volume, minute ventilation, and inspiratory flow during SB were substantially lower compared with parameters for NIV. Differences in breathing parameters may explain the lower aerosol delivery in SB. In contrast, we used the same breathing parameters for all conditions of this study.

In an in vitro study of spontaneously breathing tracheotomised adults with active exhalation, we found a substantial difference in aerosol deposition obtained with jet (6.61\%) and mesh nebulisers (17.67\%) in room air [4]. Although there is a discrepancy between the results of this study and our current research, it is important to note that we used an adult lung model with tracheostomy in the previous study. Also, the breathing parameters that were used in our previous research (tidal volume $400 \mathrm{~mL}$, respiratory rate 20 breaths per min, inspiratory:expiratory ratio 1:2) may account for the differences in our results.

Dugernier et al. [34], using single photon emission computed tomography comparison of lung deposition in six healthy subjects, reported a lung dose of $5.2 \%$ with a jet nebuliser versus $34.1 \%$ with a mesh nebuliser and chamber. Both studies report similar deposition with both nebulisers. Previously, we compared a jet and mesh nebuliser in simulated spontaneously breathing adults and found that the delivery efficiency of the jet nebuliser was 7\% while the mesh nebuliser was 35\% [7]. In contrast, the aerosol deposition obtained in our current study was $6 \%$ and $18 \%$ with a jet and mesh nebuliser, respectively. Although we used the same type of nebulisers in both projects, different flow rates were used to operate the nebulisers. For instance, the jet nebuliser was operated at $10 \mathrm{~L} \cdot \mathrm{min}^{-1}$ in our previous research as opposed to $6 \mathrm{~L} \cdot \mathrm{min}^{-1}$ in this study. Similarly, the mesh nebuliser (Aerogen Ultra) was run at $6 \mathrm{~L} \cdot \mathrm{min}^{-1}$ in the current project as opposed to no oxygen flowrate in the study by Alcoforado et al. [35]. We previously reported that the inhaled dose was two-fold higher with the mesh nebuliser and chamber without oxygen flow than with oxygen flow of $6 \mathrm{~L} \cdot \mathrm{min}^{-1}$. The simulated exhaled humidity in our model combined with differences in breathing parameters used in both projects likely contributed to the discrepancy in our results. Consequently, our findings appear to be consistent with these two in vivo studies of spontaneous breathing.

\section{Limitations and future research}

First, we used only one set of adult breathing parameters in this study that does not represent the array of ventilator settings, patterns, and modes in clinical practice. Second, only one type of jet nebuliser and mesh nebuliser was used in this research, and their performance on different modes of ventilation should not be generalised to other nebulisers within each type. Similar studies with other aerosol devices, such as pressurised metered-dose inhalers, are needed. Also, it is well known that small particles are exhaled by patients, while they are captured on the collecting filter used in bench studies that overestimate aerosol drug delivery. Therefore, in vivo, clinical studies are needed to better quantify delivered dose with various aerosol devices during MV, NIV, and SB.

In conclusion, the delivered dose distal to the trachea of an adult lung model is similar or greater with MV than NIV and SB, suggesting that artificial airway may provide a similar or better pathway for medical aerosols to the lung. Delivered dose to the adult lung model with a mesh nebuliser was greater and differed between MV, NIV, and SB, while deposition was low with a jet nebuliser and similar between the modes of ventilation tested. Further clinical studies are warranted.

Conflict of interest: A. Ari reports personal fees from Aerogen Ltd and Philips Healthcare during the conduct of the study. J.B. Fink is CSO of Aerogen Pharma Company.

\section{References}

$1 \quad$ Ari A. Effect of nebulizer type, delivery interface, and flow rate on aerosol drug delivery to spontaneously breathing pediatric and infant lung models. Pediatr Pulmonol 2019; 54: 1735-1741.

2 Ari $\mathrm{A}$, Dang $\mathrm{T}$, Al Enazi $\mathrm{FH}$, et al. Effect of heat moisture exchanger on aerosol drug delivery and airway resistance in simulated ventilator-dependent adults using jet and mesh nebulizers. J Aerosol Med Pulm Drug Deliv 2018; 31: 42-48. 
3 Ari A, Alwadeai KS, Fink JB. Effects of heat and moisture exchangers and exhaled humidity on aerosol deposition in a simulated ventilator-dependent adult lung model. Respir Care 2017; 62: 538-543.

4 Ari A, Harwood R, Sheard M, et al. Quantifying aerosol delivery in simulated spontaneously breathing patients with tracheostomy using different humidification systems with or without exhaled humidity. Respir Care 2016; 61: 600-606.

5 AlQuaimi M, Fink J, Ari A. Efficiency of aerosol devices and masks during noninvasive positive pressure ventilation in a simulated adult lung model. J Respir Med Lung Dis 2017; 2: 1018-1023.

6 Alhamad BR, Fink JB, Harwood RJ, et al. Effect of aerosol devices and administration techniques on drug delivery in a simulated spontaneously breathing pediatric tracheostomy model. Respir Care 2015; 60: 1026-1032.

7 Ari A, de Andrade AD, Sheard M, et al. Performance comparisons of jet and mesh nebulizers using different interfaces in simulated spontaneously breathing adults and children. $J$ Aerosol Med Pulm Drug Deliv 2015; 28 : 281-289.

8 Galindo-Filho VC, Ramos ME, Rattes CS, et al. Radioaerosol pulmonary deposition using mesh and jet nebulizers during noninvasive ventilation in healthy subjects. Respir Care 2015; 60: 1238-1246.

9 Lin HL, Wan GH, Chen YH, et al. Influence of nebulizer type with different pediatric aerosol masks on drug deposition in a model of a spontaneously breathing small child. Respir Care 2012; 57: 1894-1900.

10 Moody GB, Luckett PM, Shockley CM, et al. Clinical efficacy of vibrating mesh and jet nebulizers with different interfaces in pediatric subjects wiith asthma. Respir Care 2020; 65: 1451-1463.

11 Moody GB, Ari A. Quantifying continuous nebulization via high flow nasal cannula and large volume nebulizer in a pediatric model. Pediatr Pulmonol 2020; 55: 2596-2602.

12 Ari A, Areabi H, Fink JB. Evaluation of position of aerosol device in two different ventilator circuits during mechanical ventilation. Respir Care 2010; 55: 837-844.

13 Saeed $\mathrm{H}$, Mohsen M, Salah Eldin A, et al. Effects of fill volume and humidification on aerosol delivery during single-limb noninvasive ventilation. Respir Care 2018; 63: 1370-1378.

14 Saeed S, Elberry A, Eldin S, et al. Effect of nebulizer designs on aerosol delivery during noninvasive mechanical ventilation: a modeling study of in vitro data. Pulm Ther 2017; 3: 233-241.

15 ElHansy MHE, Boules ME, El Essawy AFM, et al. Inhaled salbutamol dose delivered by jet nebulizer, vibrating mesh nebulizer and metered dose inhaler with spacer during invasive mechanical ventilation. Pulm Pharmacol Ther 2017; 45: 159-163.

16 Vecellio L, Abdelrahim ME, Montharu J, et al. Disposable versus reusable jet nebulizers for cystic fibrosis treatment with tobramycin. J Cyst Fibros 2011; 10: 86-92.

17 Abdelrahim ME, Plant $\mathrm{P}$, Chrystyn $\mathrm{H}$. In-vitro characterisation of the nebulised dose during non-invasive ventilation. J Pharm Pharmacol 2010; 62: 966-972.

18 Michotte JB, Jossen E, Roeseler J, et al. In vitro comparison of five nebulizers during noninvasive ventilation: analysis of inhaled and lost doses. J Aerosol Med Pulm Drug Deliv 2014; 27: 430-440.

19 Vecellio L, De Gersem R, Le Guellec S, et al. Deposition of aerosols delivered by nasal route with jet and mesh nebulizers. Int J Pharm 2011; 407: 87-94.

20 Dugernier J, Hesse M, Jumetz T, et al. Aerosol delivery with two nebulizers through high-flow nasal cannula: a randomized cross-over single-photon emission computed tomography study. J Aerosol Med Pulm Drug Deliv 2017; 30: 349-358.

21 Rau JL, Ari A, Restrepo RD. Performance comparison of nebulizer designs: constant-output, breath-enhanced, and dosimetric. Respir Care 2004; 49: 174-179.

22 Maclntyre NR, Silver RM, Miller CW, et al. Aerosol delivery in intubated, mechanically ventilated patients. Crit Care Med 1985; 13: 81-84.

23 Fuller $\mathrm{H}$, Dolovich $\mathrm{M}$, Chambers $\mathrm{C}$, et al. Aerosol delivery during mechanical ventilation: a predictive in-vitro lung model. J Aerosol Med Pulm Drug Deliv 1992; 5: 251-259.

24 Diot P, Morra L, Smaldone GC. Albuterol delivery in a model of mechanical ventilation. Comparison of metered-dose inhaler and nebulizer efficiency. Am J Respir Crit Care Med 1995; 152: 1391-1394.

25 Fink JB, Dhand R, Grychowski J, et al. Reconciling in vitro and in vivo measurements of aerosol delivery from a metered-dose inhaler during mechanical ventilation and defining efficiency-enhancing factors. Am J Respir Crit Care Med 1999; 159: 63-68.

26 Lange C, Finlay W. Overcoming the adverse effect of humidity in aerosol delivery via pressurized metered-dose inhalers during mechanical ventilation. Am J Respir Crit Care Med 2000; 161: 1614-1618.

27 Williams JP, Ari A, Shanmugam R, et al. The effect of different closed suction catheter designs and pMDI adapters on aerosol delivery in simulated adult mechanical ventilation with and without exhaled humidity. Respir Care 2018; 63: 1154-1161.

28 Ari A, Atalay OT, Harwood R, et al. Influence of nebulizer type, position, and bias flow on aerosol drug delivery in simulated pediatric and adult lung models during mechanical ventilation. Respir Care 2010; 55: 845-851. 
29 Ari A, Roark S, Lucrecia L, et al. Influence of nasal cannula, flow rate and humidifier in aerosol drug delivery during high flow nasal oxygen administration in a simulated neonatal lung model. Respir Care 2010; 55: 1576.

30 Dugernier J, Wittebole X, Roeseler J, et al. Influence of inspiratory flow pattern and nebulizer position on aerosol delivery with a vibrating-mesh nebulizer during invasive mechanical ventilation: an in vitro analysis. J Aerosol Med Pulm Drug Deliv 2015; 28: 229-236.

31 Galindo-Filho VC, Brandao DC, Ferreira Rde C, et al. Noninvasive ventilation coupled with nebulization during asthma crises: a randomized controlled trial. Respir Care 2013; 58: 241-249.

32 Galindo-Filho VC, Alcoforado L, Rattes C, et al. A mesh nebulizer is more effective than jet nebulizer to nebulize bronchodilators during non-invasive ventilation of subjects with COPD: a randomized controlled trial with radiolabeled aerosols. Respir Med 2019; 153: 60-67.

33 Franca EE, Dornelas de Andrade AF, Cabral G, et al. Nebulization associated with bi-level noninvasive ventilation: analysis of pulmonary radioaerosol deposition. Respir Med 2006; 100: 721-728.

34 Dugernier J, Hesse M, Vanbever R, et al. SPECT-CT comparison of lung deposition using a system combining a vibrating-mesh nebulizer with a valved holding chamber and a conventional jet nebulizer: a randomized cross-over study. Pharm Res 2017; 34: 290-300.

35 Alcoforado L, Ari A, Barcelar J, et al. Impact of gas flow and humidity on trans-nasal aerosol deposition via nasal cannula in adults: a randomized cross-over study. Pharmaceutics 2019; 11: 320. 